\title{
Micro-Oscillator as Integrable Sensor for Structure-Borne Ultrasound ${ }^{\dagger}$
}

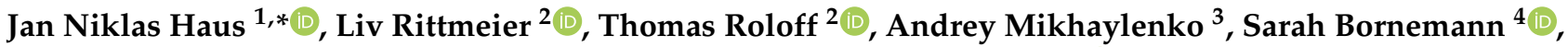 \\ Michael Sinapius ${ }^{2}$, Natalie Rauter ${ }^{3}$, Walter Lang ${ }^{4}$ and Andreas Dietzel ${ }^{1}$ (D)
}

1 Institute of Microtechnology, Technische Universität Braunschweig, 38124 Braunschweig, Germany; a.dietzel@tu-braunschweig.de

2 Institute of Mechanics and Adaptronics, Technische Universität Braunschweig, 38106 Braunschweig, Germany; 1.rittmeier@tu-braunschweig.de (L.R.); thomas.roloff@tu-braunschweig.de (T.R.); m.sinapius@tu-braunschweig.de (M.S.)

3 Institute of Mechanics, Helmut-Schmidt-Universität, 22043 Hamburg, Germany; mikhayla@hsu-hh.de (A.M.); rautern@hsu-hh.de (N.R.)

4 Institute for Microsensors, Actuators and Systems, Universität Bremen, 28359 Bremen, Germany; sbornemann@imsas.uni-bremen.de (S.B.); wlang@imsas.uni-bremen.de (W.L.)

* Correspondence: j.haus@tu-braunschweig.de; Tel.: +49-531-391-9759

+ Presented at the 8th International Electronic Conference on Sensors and Applications, 1-15 November 2021; Available online: https://ecsa-8.sciforum.net.

Citation: Haus, J.N.; Rittmeier, L.; Roloff, T.; Mikhaylenko, A.;

Bornemann, S.; Sinapius, M.; Rauter,

N.; Lang, W.; Dietzel, A. Micro-

Oscillator as Integrable Sensor for Structure-Borne Ultrasound. Eng.

Proc. 2021, 10, 81. https://doi.org/

$10.3390 /$ ecsa- $8-11313$

Academic Editor: Stefano Mariani

Published: 1 November 2021

Publisher's Note: MDPI stays neutral with regard to jurisdictional claims in published maps and institutional affiliations.

Copyright: (C) 2021 by the authors. Licensee MDPI, Basel, Switzerland. This article is an open access article distributed under the terms and conditions of the Creative Commons Attribution (CC BY) license (https:// creativecommons.org/licenses/by/ $4.0 /)$.

\begin{abstract}
Motivated by their functional conformity, micro-cantilever-based MEMS oscillators are investigated in this study as structure-integrable transducers for the acquisition of guided ultrasonic waves in fiber-metal laminates. While acceleration-sensitive oscillators are limited in their maximum frequency, the presented displacement-sensitive oscillator is operated quasi-free in the fashion of a seismometer, making it particularly sensitive for high-frequency displacements above the sensor's resonance frequency. The potential of this non-traditional application of a seismometer for the acquisition of structure-borne ultrasound is demonstrated experimentally. Therefore, MEMS oscillators are formed from the membrane of established pressure sensors by femtosecond laser micro-machining and mounted onto a setup for stimulation by structure-borne ultrasound. Experimental results indicate the targeted proportionality of the high-frequency stimulus and the sensor response. In conclusion, MEMS oscillators enable acquisition of high-frequency displacements and could therefore serve as structure-integrable sensors for guided ultrasonic waves.
\end{abstract}

Keywords: Structural Health Monitoring (SHM); Fiber Metal Laminates (FML); MEMS oscillator; ultrasound transducer; forced quasi-free oscillation; structure-integration; acoustic impedance matching; functional compliance

\section{Introduction}

For Structural Health Monitoring (SHM) of composite materials such as Carbon Fiber Reinforced Plastic (CFRP), the propagation of Guided Ultrasonic Waves (GUW) can be monitored using a network of surface-mounted piezoelectric transducers recording the local surface bending state as a function of time [1]. Any defects-presenting as cracks or delaminations in the monitored structure-result in a local change of the acoustic impedance, which causes reflections and mode-conversion of the acoustic signal, finally resulting in a change of the acoustic footprint in the sensor recordings. From this digital footprint, damages can be localized, quantified, and classified.

Modern aircraft use Fiber Metal Laminates (FML), e.g., GLARE (Glass Laminate Aluminum Reinforced Epoxy), as construction materials because they combine the favorable ductile properties of metals with the high tensile strength of composite materials [2]. A further benefit of FML is that material degradation occurs as a steady process, so that the material's condition can be monitored and quantified until service is required. 
Elastic waves in plates propagate in the form of GUW. They are characterized by velocity dispersion-which describes the dependence of the phase velocity from the signal frequency-and the material's elasticity and density. Lamb waves occur in symmetric modes and antisymmetric modes of increasing order.

In contrast to homogenous materials such as metals or CFRP, the high difference in acoustic impedance between the layers of FML, e.g., glass fiber and aluminum layers for GLARE, is expected to have an impact on the wave propagation [3].

To gain a better understanding of this complex wave propagation within FML, its inner layers need to be monitored individually. Therefore, an SHM approach for FML requires embedded sensors, which should only interfere minimally with the propagating waves.

The typical ceramic piezoelectric transducers are inappropriate because their dimensions are inevitably large and the material is poorly adapted to the acoustic impedance of the surrounding material. Suitable sensors should therefore be made of materials with better-matched acoustic impedance. Sensors that are smaller than the wavelength of the propagating ultrasound waves should interfere even less [4]. To address these requirements on the sensor, structure-integrable and impedance-matched sensors for GUW are presented in this study using MEMS oscillators.

\section{Micro-Oscillator Concept}

The acoustic impedance for GUW is calculated as follows: $Z_{f}=\rho \cdot c_{g}$, with density $\rho$ and group velocity $c_{g}$. Investigations for a frequency-thickness product of $48 \mathrm{kHz} \mathrm{mm}$ show that the acoustic impedance of typical MEMS materials such as silicon $\left(Z_{f}=20.2 \times 10^{6} \mathrm{~kg} \cdot \mathrm{m}^{-2} \cdot \mathrm{s}^{-1}\right)$ or borosilicate glass $\left(Z_{f}=11.8 \times 10^{6} \mathrm{~kg} \cdot \mathrm{m}^{-2} \cdot \mathrm{s}^{-1}\right)$ is much better adapted to glass fiber laminate $\left(Z_{f}=10.1 \times 10^{6} \mathrm{~kg} \cdot \mathrm{m}^{-2} \cdot \mathrm{s}^{-1}\right)$ than typical piezo ceramics, e.g., PZT $\left(Z_{f}=35.8 \times 10^{6} \mathrm{~kg} \cdot \mathrm{m}^{-2} \cdot \mathrm{s}^{-1}\right)$. Further, the shape and size of MEMS devices can be adapted.

A micro-mechanical oscillator consists of a spring-loaded mass that, when subjected to a force by an external acceleration, displaces relative to its frame. The displacement can be transduced (typically by capacitive or piezoresistive schemes) into an electrical sensor signal [5]. Considering the dynamics of a spring-loaded mass, a second-order model must be used to calculate the complex amplitude. The real part of this complex amplitude is given in Figure 1, where a weakly damped system is assumed as an example. As long as the excitation frequency is well below the resonance frequency, a quasi-static response with a signal amplitude proportional to the acceleration amplitude is obtained. A forced quasi-free oscillation is obtained if the excitation frequency is far above the resonance of the spring-mass system, as known for a seismograph [6]. In this regime, the sensor signal only weakly depends on the acceleration amplitude, making it unsuitable for acceleration sensing applications. However, if the displacement of the sensor frame is considered instead of acceleration, a strong and practically frequency-independent relation is obtained. For this reason, structure-borne ultrasound can be picked up as a quasi-free oscillation of a micro-cantilever or of other MEMS accelerometers. 


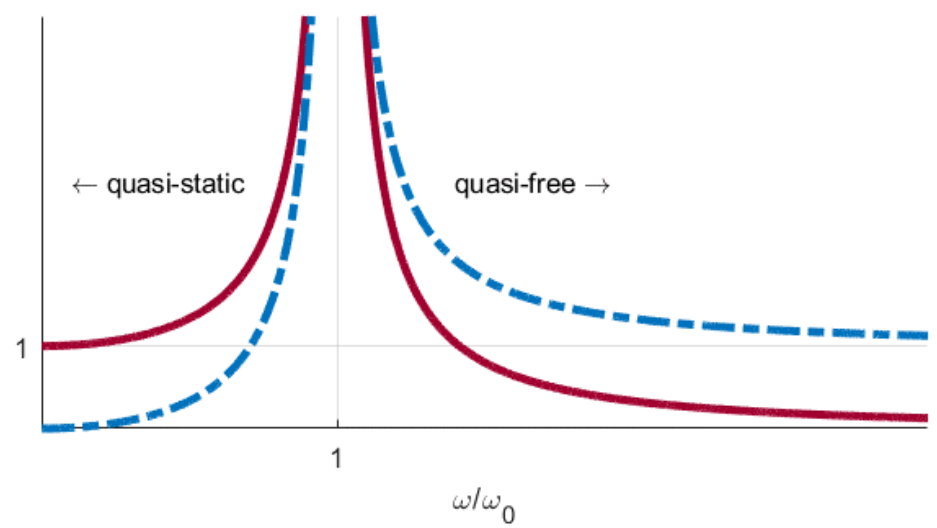

Figure 1. Signal amplitude of a generic mass-spring oscillator divided by constant external acceleration amplitude (red) compared to signal amplitude divided by constant external displacement amplitude (blue). Amplitude ratios are given in arbitrary units.

\section{Materials and Methods}

\subsection{Sensor Manufacturing}

For the proof of the quasi-free oscillation concept for structure-borne ultrasound recording, a recently developed structure-integrable pressure sensor [7] was modified to form a micro-cantilever oscillator. The unsupported silicon membrane of the pressure sensor is released from three sides, using femtosecond laser micro-machining. This yields a single crystalline micro-cantilever with piezoresistive read-out with a well-defined spring constant, which is presented in Figure 2. The signal for cantilever displacement is read out through a quarter-bridge circuit, which consists of piezoresistive paths defined by local boron doping. Four pads on the sensor's bottom side provide the terminals of the circuitry, allowing flip-chip bonding to a PCB substrate via soldering.



Figure 2. Photography of the micro-cantilever sensor mounted onto polyimide substrate (left). Microscopic bottom-view of the sensor, showing the position of the micro-cantilever in relation to the glass-cavity (middle) and the marked positions of the piezoresistors (right).

\subsection{Test Setup}

To generate an environment for ultrasonic excitation and recording, a test bed as illustrated in Figure 3 was used. For ultrasound excitation, a piezoelectric transducer was soldered onto a FR4 PCB substrate, which was adhesively bonded to a metal plate as a socket. The micro-cantilever chip was flip-chip bonded (soldered) onto a thin polyimide PCB for signal transport. This PCB was then bonded to the piezoelectric ultrasound source driven by a signal generator using a thin layer of superglue. The sensor's Wheatstone circuit was evaluated using a high-frequency bridge amplifier (DEWETRON, DAQP-BRIDGE-B) without filtering and with the amplification set to $10 \times$. The amplifier's output was recorded using one channel of a digital oscilloscope, while the excitation signal was simultaneously recorded on the second channel. 


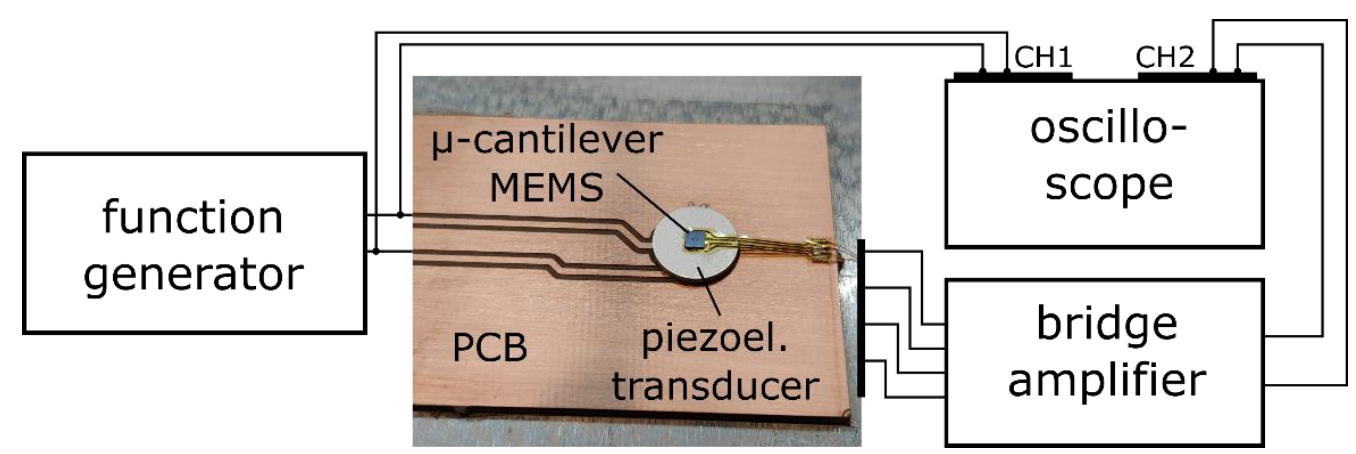

Figure 3. Test setup with piezoelectric excitement and MEMS micro-cantilever sensor.

\section{Results}

Bursts of eight sine waves with an amplitude of $10 V_{p p}$ and different frequencies were used for the out-of-plane excitation.

First, the frequency was set to $42 \mathrm{kHz}$, which is the first bending mode resonance frequency of the micro-cantilever. The transfer behavior is presented in Figure 4. In resonance, more energy is transferred into the oscillatory system than is dissipated by damping. The stored energy accumulates and the cantilever's deflection amplitude increases with each excitation cycle. In resonance, the structure-borne ultrasound waveform cannot be reconstructed easily.

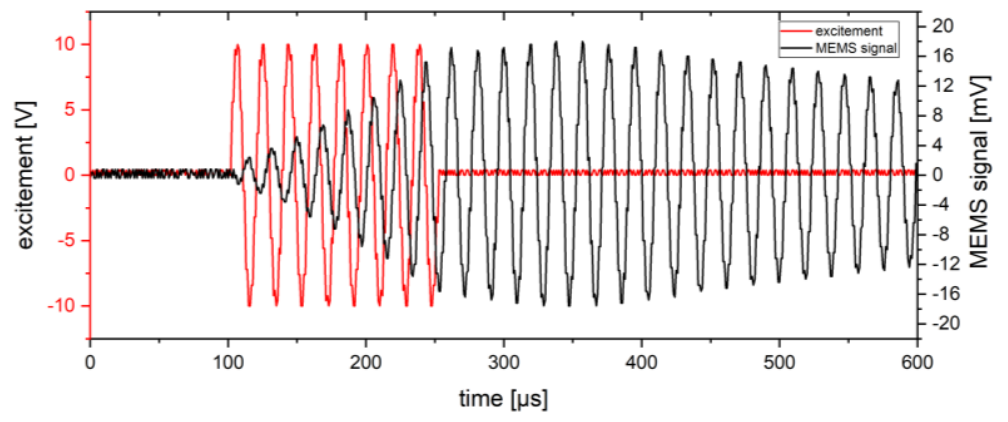

Figure 4. Transient response of the micro-cantilever sensor (black), excited with a near-resonance $42 \mathrm{kHz}$ eight-period sine burst (red).

Next, a frequency was chosen, lying between the first $(\approx 42 \mathrm{kHz})$ and second $(\approx 280 \mathrm{kHz})$ bending mode resonance frequencies of the micro-cantilever. Figure 5 shows the time response of the cantilever to a burst of $100 \mathrm{kHz}$. The obtained signal waveform is, after a short transient, proportional to that of the ultrasound.

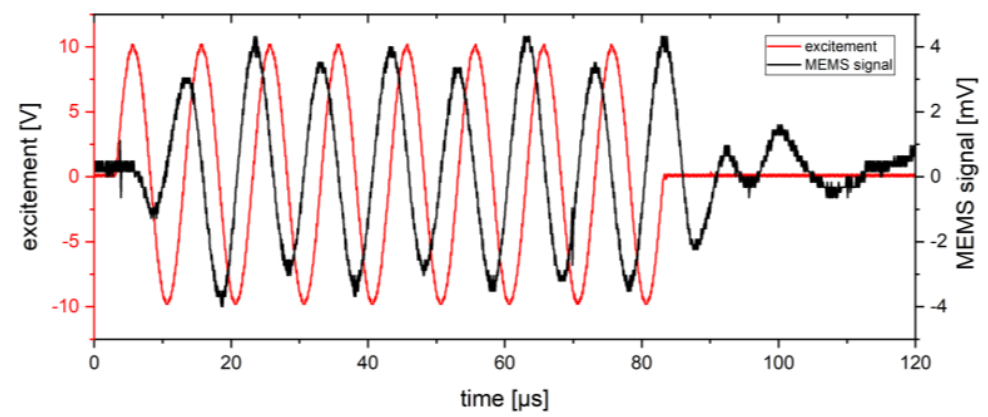

Figure 5. Transient signal response of the micro-cantilever sensor (black), excited with a $100 \mathrm{kHz}$ eight-period sine burst (red).

At frequencies below the first bending mode (resonance) frequency, the local periodic displacement was not sufficient to be extracted from the sensor signal. 


\section{Discussion and Outlook}

The presented experiment has proven the validity of the concept of structure-borne ultrasound recording using a MEMS oscillator in quasi-free excitation. The obtained sensor signal is almost proportional to the waveform of structure-borne ultrasound excitation. However, the sensor signal contains small non-linear contributions, which are probably a result of the cantilever harmonic modes, which are weakly excited by the signal's bandwidth.

In further investigations, the presented quasi-free sensor concept will be investigated in depth. Tailored sensors will improve the sensitivity and adjust the sensor's inherent dynamics; a tailored ultrasonic test setup will allow experiments with a mode-selective excitation of the sensors on an actual composite waveguide [8]. Moreover, an anodically bonded sensor lid will allow for material integration and would additionally provide a possibility for damping adaption via adjustment of the enclosed atmosphere.

Author Contributions: Conceptualization, J.N.H., L.R., A.D. and M.S.; methodology, J.N.H., T.R., L.R. and S.B.; validation, J.N.H.; formal analysis, J.N.H., A.M. and A.D.; investigation, J.N.H.; resources, S.B.; data curation, J.N.H. and A.M.; writing-original draft preparation, J.N.H.; writing-review and editing, A.D., N.R., M.S. and T.R.; visualization, J.N.H.; supervision, A.D., M.S., W.L. and N.R.; project administration, M.S.; funding acquisition, M.S., A.D., W.L. and N.R. All authors have read and agreed to the published version of the manuscript.

Funding: The authors expressly acknowledge the financial support for this research from Research Unit 3022 “Ultrasonic Monitoring of Fibre Metal Laminates Using Integrated Sensors" (Project number: 418311604) of the German Research Foundation (Deutsche Forschungsgemeinschaft (DFG)).

Institutional Review Board Statement: Not applicable.

Informed Consent Statement: Not applicable.

Data Availability Statement: The raw data of the experiments can be requested from the authors.

Conflicts of Interest: The authors declare no conflict of interest. The funders had no role in the design of the study; in the collection, analyses, or interpretation of data; in the writing of the manuscript; or in the decision to publish the results.

\section{References}

1. Balageas, G.; Fritzen, C.P.; Güemes, A. Structural Health Monitoring; ISTE Ltd.: London, UK, 2006.

2. Chai, G.B.; Manikandan, P. Low velocity impact response of fibre-metal laminates-A review. Compos. Struct. 2014, 107, 363-381. [CrossRef]

3. Rittmeier, L.; Losch, T.; Sinapius, M.; Lammering, R. Investigation on the influence of material interfaces and impedance changes on the propagation of guided waves in laminated steel layers. Procedia Manuf. 2018, 24, 196-202. [CrossRef]

4. Lammering, R.; Gabbert, U.; Sinapius, M.; Schuster, T.; Wierach, P. (Eds.) Lamb-Wave Based Structural Health Monitoring in Polymer Composites; Springer: Berlin/Heidelberg, Germany, 2017; p. 454.

5. Büttgenbach, S.; Constantinou, I.; Dietzel, A.; Leester-Schädel, M. Case Studies in Micromechatronics; Springer: Berlin/Heidelberg, Germany, 2020.

6. Younis, M.I. MEMS Linear and Nonlinear Statics and Dynamics; Springer Science \& Business Media: Berlin/Heidelberg, Germany, 2011; ISBN 9781441960207.

7. Haus, J.N.; Schwerter, M.; Schneider, M.; Gäding, M.; Leester-Schädel, M.; Schmid, U.; Dietzel, A. Robust Pressure Sensor in SOI Technology with Butterfly Wiring for Airfoil Integration. Sensors 2021, 21, 6140. [CrossRef] [PubMed]

8. Rittmeier, L.; Roloff, T.; Haus, J.; Dietzel, A.; Sinapius, M. Design of a Characterization Environment for a MEMS Ultrasound Sensor under Guided Ultrasonic Wave Excitation. In Proceedings of the 8th International Electronic Conference on Sensors and Applications, Basel, Switzerland, 1-15 November 2021. [CrossRef] 